\title{
Éditorial
}

\section{Un retour à l'école}

Au moment où en France, comme dans la plupart des pays occidentaux, on voit depuis quelques années nos étudiants bouder les études scientifiques universitaires, on ne peut que se réjouir de voir un retour de l'apprentissage de la radioactivité au lycée.

Cette bonne nouvelle, nous la devons à nos amis Marie-Christine Robé, Claude Bertrand et Bernard Bonin qui ont proposé, avec l'aide de leur organisation respective (IRSN, ALGADE et COGEMA), un système simple pour mesurer le radon. Sa description apparaît dans nos colonnes sous la rubrique «produits nouveaux » qu'anime notre président émérite Daniel Blanc. L'utilisation de ce kit sera au programme des terminales ; remercions l'Éducation nationale qui a réparé une lacune que nous déplorions tous.

C'est aussi le moment de rappeler à cette honorable institution que la SFRP a déjà agi dans ce sens, la plaquette « La radioactivité naturelle en 10 épisodes » est un complément parfait à ce kit, elle est toujours disponible au secrétariat de la SFRP, qu'on se le dise.

Ce concept de « radioactivité naturelle » reprend fort heureusement tout son sens au moment où la CIPR propose pour le futur de s'y référer pour bâtir un système de protection radiologique mieux compris par le public.

Par ailleurs, j'ai le plaisir de vous annoncer l'arrivée de trois nouveaux membres dans le comité de rédaction de la revue. En effet, Philippe Bérard (CEA), Pascal Crouail (CEPN) et François Paquet (IRSN) nous rejoignent, le Comité leur souhaite la bienvenue. Pris par d'autres tâches, Christian Lefaure nous quitte, un grand merci pour toute son action pour la revue, j'espère qu'il continuera à travailler pour nous. Nous avons choisi un nouveau vice-président, ce sera une vice-présidente puisque le Comité, à l'unanimité, a désigné notre amie Jeannine Lallemand pour ce poste. Je ne peux que m'en réjouir.

Ce début d'année 2003 est donc dans la continuité de 2002, où nous avons renouvelé le panel de nos correspondants étrangers. Déjà dans ce numéro, l'un d'eux Christophe Murith nous fait part d'une information importante venant de Suisse... C'est bien parti.

À très bientôt,

Henri Métivier

Président du comité de la revue 